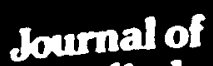 Applied Mechanics

A Brief Note is a short paper that presents a specific solution of technical interest in mechanics but which does not necessarily contain new general methods or results. A Brief Note should not exceed 1500 words or equivalent (a typical one-column figure or table is equivalent to $\mathbf{2 5 0}$ words; a one line equation to 30 words). Brief Notes will be subject to the usual review procedures prior to publication. After approval such Notes will be published as soon as possible. The Notes should be submitted to the Technical Editor of the Journal of A pplied Mechanics. Discussions on the Brief Notes should be addressed to the Editorial Department, ASME, United Engineering Center, 345 East 47th Street, New York, N. Y. 10017, or to the Technical Editor of the Journal of A PPLIED Mechanics. Discussions on Brief Notes appearing in this issue will be accepted until two months after publication. Readers who need more time to prepare a Discussion should request an extension of the deadline from the Editorial Department.

\section{Inelastic Post-Buckling of Columns}

\section{G. Prathap ${ }^{1}$ and T. K. Varadan ${ }^{2}$}

\section{Introduction}

Slender cantilever columns find extensive use as struts carrying compressive loads. In the optimum design of these members, it is necessary to take into account both geometrical and physical nonlinearities in the analysis. Oden and Childs (1970) studied the post-buckling of a nonlinearly elastic bar of uniform cross-section with a compressive tip load by assuming a moment-curvature relationship which simulated that of a class of elasto-plastic material. Verma and Krishnamurty (1974) studied the post-buckling problem for tapered beams of rectangular cross section incorporating material nonlinearity in terms of Ramberg-Osgood stress-strain relations (Ramberg and Osgood, 1943) using a finite-difference approach with reduced modulus concept. Recently, Monasa (1974, 1977) studied the large deformation of very slender elasto-plastic bars using a nonlinear moment-curvature relationship (Beedle, 1966)to represent the behavior in the plastic zone. The important assumption in the investigation by Monasa $(1974,1977)$ is that the axial stress is negligible compared to bending stress and may be neglected.

The concept of integrating through the thickness to account for the stress reversal has been extensively applied before and it is the common practice. In this Note, the earlier procedure of integrating through the thickness as indicated by the present authors (1976), is used to determine the axial load and bending moment that each section can carry, and this is further used to compute the state of deformation in the structure. This indirect numerical iterative analysis allows the post-buckling behavior of a structure for nonlinearly elastic material to be

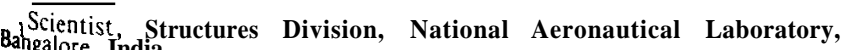
agalore, India

Professor, Department of Aeronautical Engineering, Indian Institute of Technology, Madras, India.

Manuscript received by ASME Applied Mechanics Division, August 31, 1984,
thal revision
}

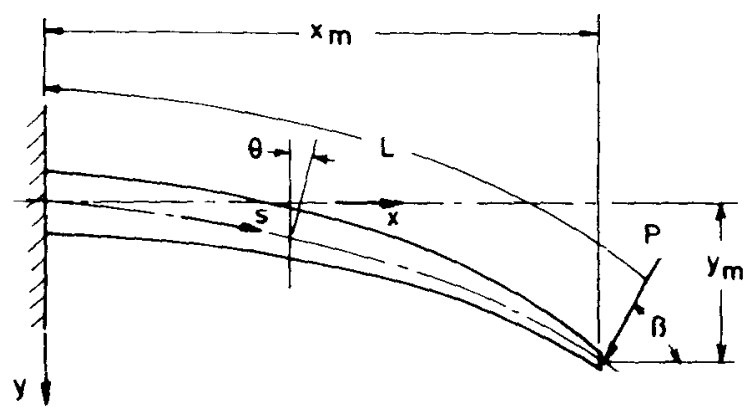

Fig. 1 Cantilever column: coordinate system

determined as function of the parameters in the stress-strain laws.

\section{Analysis}

The deformed configuration and the coordinate system used here for the cantilever column with an inclined tip load is shown in Fig. 1. A class of columns of variable rectangular cross-section, of depth $D(s)$ and breadth $B(s)$ are chosen in the form $D(s)=D_{o} d(s)$ and $B(s)=B_{0} b(s)$. The Ramberg-Osgood relation (Ramberg and Osgood, 1943) is rewritten in an alternate form similar to that suggested by Rao and Krishnamurty (1971) as

$$
\left(\sigma / \sigma_{r}\right)=f(\epsilon)=\left[\left(\epsilon / \epsilon_{r}\right)-0.4\left(\epsilon / \epsilon_{r}\right)^{m}\right]
$$

where $\sigma_{r}, \epsilon_{r}, m$ are parameters that determine the nonlinearity, and $\sigma, \epsilon$ are the stress and strain, respectively. In a deflected stable equilibrium position, the total compressive stress is

$$
\sigma_{o}(\eta)=P \cos (\beta+\theta) /\left(B_{o} D_{o} b d\right)
$$

and bending moment is

$$
M(\eta)=P\left[\left(x_{m}-x\right) \sin \beta+\left(y_{m}-y\right) \cos \beta\right] .
$$

Normal force and bending moment resisted by the section are

$$
B_{o} D_{o} b d \sigma_{r} \int_{-\alpha_{1}}^{\alpha_{2}} f\left(\epsilon_{o}+\lambda \alpha\right) d \alpha \text { and }
$$

$$
B_{o} D_{o}^{2} b d^{2} \sigma_{r} \int_{-\alpha_{1}}^{\alpha_{2}} f\left(\epsilon_{o}+\lambda \alpha\right) \alpha d \alpha, \text { respectively. }
$$

Here $\lambda=(D / L)(d \theta / d \eta), \eta=s / L, \alpha=(z / D)$, where $z$ is the distance measured from neutral axis. We know from equa- 


$$
\frac{\sigma_{o}(\eta)}{\sigma_{r}}=f\left(\epsilon_{o}\right)=\frac{P}{\sigma_{,} B_{o} D_{o}} \frac{\cos (\beta+\theta)}{b d}
$$

From equilibrium consideration, we have from above equations

$$
\int_{-\alpha_{1}}^{\alpha_{2}} f\left(\epsilon_{o}+\lambda \alpha\right) d \alpha=\frac{P}{\sigma_{r} B, D,} \frac{\cos (\beta+\theta)}{b d}
$$

and

$$
\begin{aligned}
\int_{-\alpha_{1}}^{\alpha_{2}} f\left(\epsilon_{o}+\lambda \alpha\right) \alpha d \alpha & =\frac{P L}{\sigma_{r} B_{o} D_{o}^{2}} \\
& {\left[\left(\frac{x_{m}}{L}-\frac{x}{L}\right) \sin \beta+\left(\frac{y_{m}}{L}-\frac{y}{L}\right) \cos \beta\right] }
\end{aligned}
$$$$
b d^{2}
$$

Following the procedure given by Verma and Krishnamurty (1974) and defining $f(\epsilon)=\left(\epsilon / \epsilon_{r}\right)-0.4\left(\epsilon / \epsilon_{r}\right)^{m}$ in the loading or concave side of the beam, and $f(\epsilon)=\left(\epsilon / \epsilon_{r}\right)-0.4\left(\epsilon_{o} / \epsilon_{r}\right)^{m}$ in-the unloading or convex side, one obtains after some algebra, the equation

$$
\begin{array}{r}
\int_{-\alpha_{1}}^{o}\left[\left(\frac{\epsilon_{o}+\lambda \alpha}{\epsilon_{r}}\right)-0.4\left(\frac{\epsilon_{o}}{\epsilon_{r}}\right)^{m}\right] d \alpha+\int_{o}^{\alpha_{2}}\left[\left(\frac{\epsilon_{o}+\lambda \alpha}{\epsilon_{r}}\right)\right. \\
\left.-0.4\left(\frac{\epsilon_{o}+\lambda \alpha}{\epsilon_{r}}\right)^{m}\right] d \alpha=\frac{P}{\sigma_{r} B_{o} D_{o}} \frac{\cos (\beta+\theta)}{b d}
\end{array}
$$

Equation (3) may be rewritten as

$$
\begin{aligned}
\int_{-\alpha_{1}}^{\alpha_{2}} f\left(\epsilon_{o}\right) d \alpha & =\int_{-\alpha_{1}}^{\alpha_{2}}\left[-\frac{\epsilon_{o}}{\epsilon_{r}}-0.4\left(-\frac{\epsilon_{o}}{\epsilon_{r}}\right)^{m}\right] d \alpha \\
& =\frac{P}{a, B, D_{o}} \frac{\cos (\beta+\theta)}{b d}
\end{aligned}
$$

From equations (6) and (7), we obtain

$$
\frac{\lambda}{\epsilon_{r}} \int_{-\alpha_{1}}^{\alpha_{2}} \alpha d \alpha+\int_{o}^{\alpha_{2}} 0.4\left[\left(\frac{\epsilon_{o}}{\epsilon_{r}}\right)^{m}-\left(\frac{\epsilon_{-}+\lambda \alpha}{\epsilon_{r}}\right)^{m}\right] d \alpha=0
$$

Noting that $\alpha_{1}+\alpha_{2}=\mathrm{I}$, the above equation can be rewritten as $\lambda\left(1-2 \alpha_{2}\right)=\int_{0}^{\alpha_{2}}$ 0.46, $\left[\left(\frac{\epsilon_{o}}{\epsilon_{r}}\right)^{m}-\left(\begin{array}{c}\epsilon_{o}+\lambda \alpha \\ \epsilon_{\epsilon_{r}}+\lambda\end{array}\right)^{m}\right] d \alpha$

Equation (8) determines the location of the neutral axis. For the linear case, the term on the right hand side vanishes, so that $\alpha_{2}=0.5$. We therefore get an implicit nonlinear equation in $\alpha_{2}$ as

$$
\begin{aligned}
\alpha_{2}=0.5 & -\frac{0.4 \epsilon_{r}}{\lambda}\left\{\left(\frac{\epsilon_{o}}{\epsilon_{r}}\right)^{m} \alpha_{2}\right. \\
& -X(m+1) \\
& \left.-\epsilon_{r}\left[\left(\frac{\epsilon_{o}+\lambda \alpha_{2}}{\epsilon_{-}}\right)^{m+1}-\left(\frac{\epsilon_{o}}{\epsilon_{-}}\right)^{m+1}\right]\right\}
\end{aligned}
$$

The moment-curvature relationship is now established from equation (5) as

$$
\begin{aligned}
& \int_{-\alpha_{1}}^{o}\left[\left(\frac{\epsilon_{o}+\lambda \alpha}{\epsilon_{r}}\right)-0.4\left(\frac{\epsilon_{o}}{\epsilon_{r}}\right)^{m}\right] \alpha d \alpha \\
& \quad+\int_{o}^{\alpha_{2}}\left(\frac{\epsilon_{o}+\lambda \alpha}{\epsilon_{r}}\right)-0.4\left(\begin{array}{c}
\epsilon_{o}+\lambda \alpha \\
\epsilon_{r}^{+\lambda \alpha}
\end{array}\right)^{m} \alpha d \alpha \\
& =\frac{P L}{\sigma_{r} B_{o} D_{o}^{2}}\left[\frac{\left(\frac{x_{m}}{L}-\frac{x}{L}\right) \sin \beta+\left(\frac{y_{m}}{L}-\frac{y}{L}\right) \cos \beta}{b d^{2}}\right]
\end{aligned}
$$

which gives

$$
\begin{gathered}
\int_{-\alpha_{1}}^{\alpha_{2}}\left[\frac{\epsilon_{o}}{\epsilon_{r}}-0.4\left(\frac{\epsilon_{o}}{\epsilon_{r}}\right)^{m}\right] \alpha d \alpha+\frac{\lambda}{3 \epsilon_{r}}\left(\alpha_{2}^{3}+\alpha_{1}^{3}\right) \\
\quad+\int_{o}^{\alpha_{2}}\left[0.4\left(\frac{\epsilon_{o}}{\epsilon_{r}}\right)^{m}-0.4\left(\frac{\epsilon_{o}+\lambda \alpha}{\epsilon_{r}}\right)^{m}\right] \alpha d \alpha \\
=\frac{P L}{\sigma_{r} B_{o} D_{o}^{2}}\left[\frac{\left(\frac{\cdots m}{L}-\frac{\cdots}{L}\right) \sin \beta+\left(\frac{r m}{L}-\frac{r}{L}\right) \cos \beta}{b d^{2}}\right]
\end{gathered}
$$

Substituting the result from equation (7) in equation (11) again, we get

$$
\begin{array}{r}
\frac{P}{\sigma_{r} B, D_{o}} \frac{\cos (\beta+\theta)}{b d} \frac{\left(\alpha_{2}^{2}-\alpha_{1}^{2}\right)}{2}+\frac{x}{3 \epsilon_{r}}\left(\alpha_{2}^{3}+\alpha_{1}^{3}\right) \\
\mathbf{t} \int_{o}^{\alpha_{2}}\left[0.4\left(\frac{\epsilon_{o}}{\epsilon_{r}}\right)^{m}-0.4\left(\frac{\epsilon_{o}+\lambda \alpha}{\epsilon_{r}}\right)^{m}\right] \alpha d \alpha \\
=\frac{P L}{\sigma_{o} B_{o} D_{o}^{2}}\left[\frac{\left(\frac{x_{m}}{L}-\frac{x}{L}\right) \sin \beta+\left(\frac{y_{m}}{L}-\frac{y}{L}\right) \cos \beta}{b d^{2}}\right]
\end{array}
$$

Thus equations (9) and (12) together with the relation $\alpha_{1}+\alpha_{2}=\mathbf{1 . 0}$ completely define the problem.

\section{Solution}

The problem on hand is stated as follows. Give the tip load in a nondimensional form, say $\left(P L / \sigma_{r} B_{o} D_{o}^{2}\right)$ and the geometry of the beam $b(\eta), d(\eta), D_{o} / L$, and the material properties $\epsilon_{r}, m$ and $\sigma_{r}$, it is required to determine the shape of the deformed configuration. Indirect numerical iterative scheme seems to be a simple, effective method of solution and is used here as described below.

The deformed configuration is prescribed initially by considering a certain value for the end slope $\theta_{1}$. The slope $\theta(\eta)$ is assumed as $\theta_{1} \sin (\pi \eta / 2)$, satisfying the conditions $\theta(0)=0$ and $\theta(1)=\theta_{1}$. Thus, initially the value of $d \theta(\eta) / d \eta$ is equal to $\left(\pi \theta_{1} / 2\right) \cos (\pi \eta / 2) . \theta(\eta)$, and hence $x(\eta), y(\eta)$ given by the expressions

$$
x(\eta)=\int_{0}^{\eta} \cos \theta d \eta \text { and } y(\eta)=\int_{0}^{\eta} \sin \theta d \eta
$$

are calculated by numerical integration. It is known that $\lambda(\eta) / \epsilon_{r}$ is equal to $\left(D_{o} d / L \epsilon_{r}\right)(d \theta / d \eta)$ and so $\lambda(\eta) / \epsilon_{r}$ can be determined for the initial assumed value of $d \theta / d \eta$. The linear stress-strain approximation to equation (12), at the fixed end, gives

$$
\frac{\lambda(o)}{12 \epsilon_{r}}=\left(\frac{P L}{\sigma_{r} B_{o} D_{0}^{2}}\right)\left[\frac{\frac{x_{m}}{L} \sin \beta+\frac{y_{m}}{L} \cos \beta}{b d^{2}}\right]
$$

As $\lambda(o) / \epsilon_{r}$ is known, the above equation allows to determine the value of $\left(P L / \sigma_{r} B_{0} D_{o}^{2}\right)$. From this value of $\left(P L / \sigma_{r} B_{0} D_{0}^{2}\right)$, $\left(P / \sigma_{r} B_{o} D_{o}\right)$ is determined and then used in equation (3) to evaluate $\sigma_{o}(\eta) / \sigma_{r}$ and hence $\epsilon_{o} / \epsilon_{r}$ at each cross-section from equation (1). With these values of $\epsilon_{o} / \epsilon_{r}$ and $\lambda(\eta) / \epsilon_{r}, \alpha_{2}(\eta)$ is calculated at each station by iteration from equation (9). Substituting the above values in equation (12) and then carrying out the integration through thickness, first as improved value of $P L / \sigma_{r} B_{o} D_{o}^{2}$ is obtained considering $\eta=0$, using 
Table 1 Numerical values at the commencement of instability $(L / D=100)$

\begin{tabular}{lcc}
$m$ & $\left(\epsilon_{\mathrm{c}} L / D\right)$ & $12\left(\epsilon_{f} L / D\right)\left(\sigma_{a} L / \sigma_{r} D\right)$ \\
\hdashline 2 & 0.49 & 2.118 \\
3 & 0.75 & 2.420 \\
4 & 0.86 & 2.581 \\
5 & 0.92 & 2.686
\end{tabular}

\begin{tabular}{lcc}
$\left(y_{m} / L\right)$ & $\left(x_{m} / L\right)$ & $\theta_{\mathrm{L}}$ \\
$\mathbf{0 . 3 3 0}$ & 0.932 & $75 \mathrm{deg}$ \\
$\mathbf{0 . 4 8 4}$ & 0.843 & $75 \mathrm{deg}$ \\
0.558 & $\mathbf{0 . 7 8 1}$ & $75 \mathrm{deg}$ \\
0.598 & 0.740 & $75 \mathrm{deg}$ \\
\hline
\end{tabular}

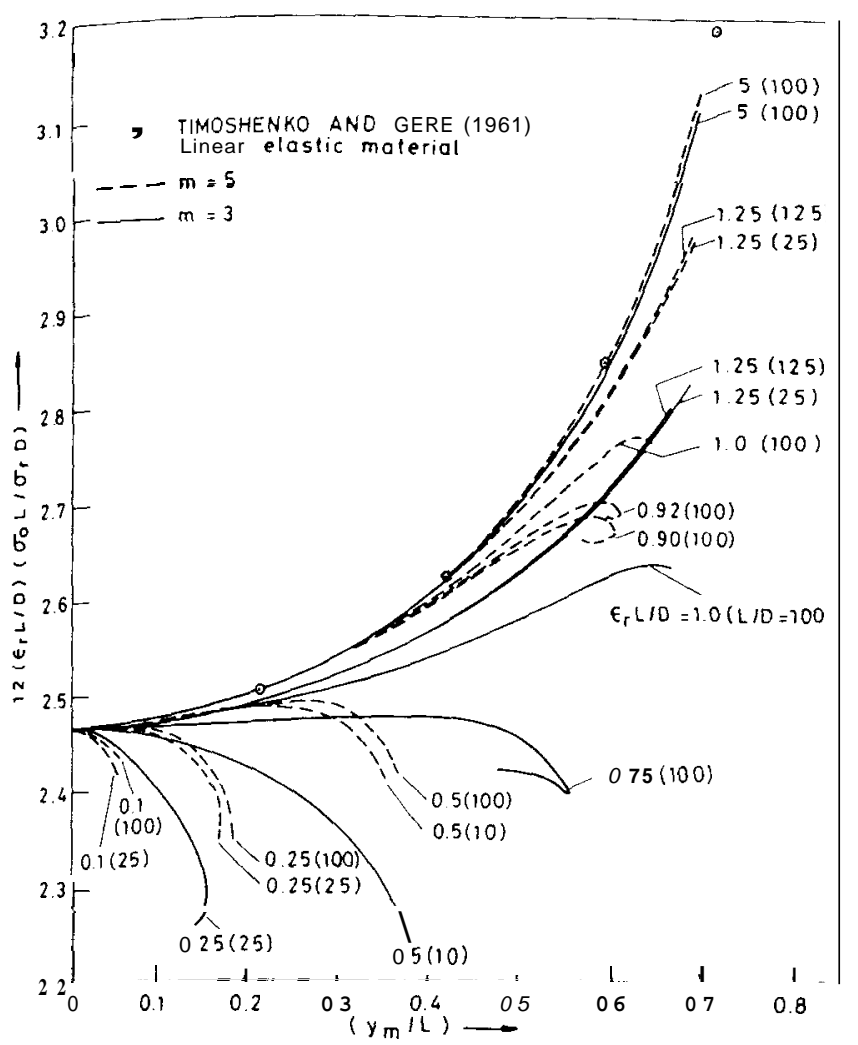

Fig. 2 Load-max. deflection relation

$\lambda(\eta) / \epsilon_{r}$ is computed from equation (12). The iteration is carried till the values of $P L / \sigma_{r} B_{0} D_{\mathrm{o}}^{2}$ and $\lambda(\eta) / \epsilon_{r}$ converge to the required degree of accuracy.

\section{Results and Discussions}

A computer algorithm is written to carry out the numerical integration and iteration for any tapered beam with a load of arbitrary inclination $\beta$. However, only results for a uniform bem $(b=d=1)$ with an axial compressive load $(\beta=0)$ are presented. The Parameters governing the nondimensional tip deflection $\left(y_{m} / L\right)$ and $\left(x_{m} / L\right)$ to nondimensional load $\epsilon_{r}\left(\sigma_{o} / \sigma_{r}\right)\left(L / D_{o}\right)^{2}$ are $\left(L / D_{o}\right), \epsilon_{r}$ and $\boldsymbol{m}$. (Note $\left(\sigma_{o} / \sigma_{r}\right)$ $\left.(L I D)=,P L / \sigma_{r} B_{o} D_{o}^{2}\right)$. For a linear problem, where $\sigma_{r} / \epsilon_{r}=\boldsymbol{E}$, we have $P L^{2} / E I_{o}=12 \sigma_{o} L^{2} / E D_{o}^{2}=\pi^{2} / 4$.

The load-maximum vertical deflection is plotted in Fig. 2 in the nondimensional form. It is found that the nondimensional plots of tip deflection-load is nearly identical for larger values of $(\epsilon, L / D)=$ constant $(>1.0)$ for each $m$. This behavior corroborates the findings of Monasa (1977) that the same family of curves can be used for different parameters $\left(\sigma_{y} / E\right)$ and $(L / D)$ provided the product $\left(\sigma_{y} L / E D\right)$ is the same. Therefore, only two nondimensional parameters, $m$ and $\left(\epsilon_{r} L / D\right)$ need be used to describe the problem when $\left(\epsilon_{r} L / D\right)>1.0$. However, from the numerical results obtained, it could be mentioned that there is a definite deviation in the behavior for smaller values of $\left(\epsilon_{r} L / D\right)=$ constant $(<0.5)$ as shown in Fig. 2, which necessitates the use of all the three parameters.

As one expects, within the range of values considered, the decrease in $m$ increases the material nonlinearity effect, as does the decrease in $\epsilon_{r}$ and $L / D$. Thus, the material non inearity effect reduces at large slenderness ratios and the geometrical nonlinearity effect predominates. This behavior is similar to that obtained for the case of inelastic laroe deforma- tion of beams under a vertical tip load (Prathap and Varadan, 1976).

Computations in each case became unstable when there was a reversal in sign of stress (The stresses are based on $\lambda \alpha$ and $\epsilon_{0}$. When the maximum bending stress computed from the maximum occurring value of $h a$ is greater than the axial stress based on $\epsilon_{0}$, the reversal of sign takes place and there will be a computational difficulty) and were terminated at that stage. The present analysis considered the tip angle up to $80 \mathrm{deg}$ in steps of $5 \mathrm{deg}$. The values of $\left(\epsilon_{r} L / D\right)$ at which instability started occurring are given in Table 1 for different values of $m$ and for a constant value of $L / D(=100)$, and this value of $\left(\epsilon_{r} L / D\right)$ decreases with the decrease in $\boldsymbol{m}$. This means that the material nonlinearity and geometrical nonlinearity play an important role in the stability behavior, the instability occurring due to the shifting of neutral axis out of the domain of the beam. From the numerical results obtained, it is noticed that increase in deflection takes place for decrease in load before the commencement of the instability of the structural member.

\section{References}

Beedle, L. S.. 1966, Plastic Design of Steel Frames, Wiley, New York, pp. 25-30.

Monasa, F. E., 1974, "Deflections and Stability Behavior of Elasto-Plastic Flexible Bars," ASME Journal of Applied Mechanic : Vol 41 pp 537538

Monasa, F. E., 1977, "'Numerical Procedure for the i) the fron of Buckled Elasto-Plastic Flexible Bars," Computers and Structures, Vol. 7, pp. 453-460.

Oden, J. T., and Childs, S. B., 1970, "Finite Deflection of a Nonlinearly Elastic Bar," ASME Journal of ApPlied Mechanics, Vol. 37, pp. 48-52.

Prathap, G., and Varadan, T. K., 1976, "The Inelastic Large Deformation of Beams,"' ASME Journal of Applied Mechanics, Vol. 43, pp. 689-690.

Ramberg, W., and Osgood, W. R., 1943, "Description of Stress-Strain Curves of Three Parameters," NACA TN 902

Rao, G. V., and Krishnamurty, A. V., 1971, "An Alternate Form of the Ramberg-Osgood Formula for Matrix-Displacement Analysis," Nuclear Engineering and Design, Vol. 17, pp. 297-308.

Timoshenko, S. P., and Cerc, J. M., 1961, Theory of Elastic Stability. McGraw Hill, New York, pp. 76-80.

Verma, N. K., and Krishnamurty, A. V., 1974, "Inelastic Post-Buckling of Columns of Variable Flexural Rigidity," Journal of StructuralMechanics, Vol. 3, pp. 77-96.

\section{Stresses Around Two Rigid Cylindrical In- clusions in an Infinite Elastic Body Under Tension}

\section{S. Itou ${ }^{3}$}

\section{Introduction}

Recently, fiber reinforced plastics have been widely used in designing the various members of a machine or structure because they are of high strength and are not heavy. In such materials, the loads are transmitted from the matrix, with a low degree of stiffness, to the fibers, with a high degree of stiffness, through the interfaces.

It is very complicated to obtain the stress field around a finite length circular cylindrical inclusion in an infinite elastic body. To bypass this difficulty, Kasano et al. (1981) assumed

\footnotetext{
${ }^{3}$ Associate Professor, Department of Mechanical Engineering, Hachinohe Institute of Technology, Hachinohe, Japan 031.

Manuscript received by ASME Applied Mechanics Division, August 19, 1985; final tarising In......... ne inos
} 